\title{
Association of Age with Hand Grip Strength, Pinch Strength and Reaction Time in Gynaecologists and Obstetricians
}

\author{
Roopa Desai ${ }^{1}$, Jagruti Maniar ${ }^{2}$, Shilpa Khandare ${ }^{3}$, Priyanka Dogra ${ }^{4}$, \\ Rucha Choudhary ${ }^{5}$ \\ ${ }^{1}$ Associate Professor, Dr. D. Y. Patil College of Physiotherapy, Dr. D. Y. Patil Vidyapeeth, Pimpri, Pune \\ ${ }^{2}$ Assistant Physiotherapist, Orthopaedics Clinic, Mumbai. \\ ${ }^{3}$ Professor, Dr. D. Y. Patil College of Physiotherapy, Dr. D. Y. Patil Vidyapeeth, Pimpri, Pune \\ ${ }^{4,5}$ Post Graduate Student, Dr. D. Y. Patil College of Physiotherapy, Dr. D. Y. Patil Vidyapeeth, Pimpri, Pune.
}

Corresponding Author: Roopa Desai

\begin{abstract}
According to scientific literature, evidence of relationship exists between dexterity and age. With increasing age, the performances get slower, less smooth, less coordinated and less controlled. Lesser the reaction time, better is the achievement seen sports, music, driving, academics and also in day-today activities. We can always predict reacting abilities of an individual based on his reaction time. In case of surgeons, this helps us to identify the surgeons with prolonged reaction time and identify the cause. Some studies suggest that there is direct relationship that exists between muscle strength and increased age.
\end{abstract}

Aim: This study aimed in assessing the association of age with hand grip strength, pinch strength and reaction time in Gynaecologists and Obstetricians.

Methods: This study involved 30 Gynaecologists and Obstetricians. Hand grip strength, Pinch Grip Strength and Reaction time were assessed on both dominant and non- dominant hand.

Results: The result showed positive correlation of age with hand grip strength and pinch strength and negative correlation of age with reaction in Gynaecologists and Obstetricians.

Conclusion: There is low association of age with hand grip strength and pinch strength, there is reduction in reaction time as age increases in Gynaecologists and Obstetricians.

Keywords: Age, Hand grip strength, Pinch Grip Strength, Reaction time, Gynecologists

\section{INTRODUCTION}

Surgeons have long duration of work and have physically demanding job. They are also exposed to cumulative awkward positions, repetitive motions and forceful exertion while performing surgery. These lead to muscular strain and development of musculoskeletal disorders. Moreover, the operating room is a complex environment where the patient's priority is first. Performing surgery involves well-known risk for developing multisite pain. The article states that there is prevalence of 93\% of musculoskeletal pain in surgeons ${ }^{1}$.
Gynaecologist have multisite musculoskeletal disorder such as lower back, neck, shoulder, upper back and wrist/hand. Performing surgery worsens the pain ranging from $76.3 \%$ to $83.7 \%{ }^{2}$ The article states that the prevalence of wrist/hand pain in gynaecologic surgeons in $60.9 \%{ }^{2}$ The hand is the complex organ with several functions. As a grip organ it is both able to exert strength and hold delicate objects; as a tactile organ, it links the body to the environment. Hand grip include power grip and pinch grip. Power grip requires firm control and gives greater 
Roopa Desai et.al. Association of age with hand grip strength, pinch strength and reaction time in gynaecologists and obstetricians.

flexor asymmetry to hand. During power grip the ulnar side of the hand work with the radial side to give the stronger stability. The ulnar digits work together to provide and static support. This grip is used whenever strength or the force is the primary consideration. Pinch grip or Precision is an activity limited to metacarpophalangeal joints and involves primarily the radial side of the hand. Precision grips, the thumb and the fingers are used and the palm may or may not be involved, there is pulp to pulp contact between the thumb and fingers and the thumb opposes the fingers. The intrinsic muscles are more important in precision than power grip, because they provide stability and control of direction and acts as buttress providing power to grip. Hand grip strength is one of the basic and important components in study of handling strength and the movement abilities of hand. It is also essential component for performing the activity of daily living, being used in $45 \%$ of the daily activity.

Grip strength is also important in gynaecologist as it is used to conduct various surgical tasks. These tasks include puncture, dissecting, probing, ligation, stapling, grasping, tying knot, suturing. There can be decrease in the hand grip strength and pinch grip with repetitive movements, or by overuse of hand muscles or injury, muscle exhaustion, pressure and neural injury and rapid fatigue caused during instrument handling. The instrument handles can affect hand strength, thus speeding up the fatigue of the hand muscles. 3 The portable Jamar dynamometer is used to test power grip strength and Pinch meter/ gauge is used to test pinch strength. Portable dynamometer measures strength that is valid, reliable and accurate. Reaction time is the delay between presence of stimulus and beginning of response. It is a measure of how quickly one can respond to particular stimulus. Lesser the reaction time, quicker is the achievement seen in day to day activities. ${ }^{4}$ In case of surgeons, this helps us to identify the surgeons with prolonged reaction time and identify the cause.

\section{MATERIAL AND METHOD}

The observational study was carried out at Dr. D.Y. Patil hospital, Pune, Dr. D.Y. Patil Ayurveda Hospital and Research Centre. Total of 30 Gynaecologists and Obstetricians were assessed with minimum 2 years of clinical experience and in an age group of 30-44 years. Practitioners with recent injuries in upper extremity were excluded from the study. The assessment of Hand grip strength was done by using Hand Held Dynamometer, with the subjects seated in upright position with feet fully resting on the floor, hip and knee positioned at 90degree, elbow was positioned on the armrest at approximately 90- degree and shoulder of the gripping arm was maintained in adduction. The Dynamometer was held with the index finger at the top of the grip, while keeping rest all the fingers in the grip band. The Dynamometer handle position was used and subjects were instructed to produce smooth gripping force without jerking movements. Mean of 3 repetitions were considered. Assessment of the pinch strength was done that included lateral pinch and pulp to pulp. This was done by using pinch meter, the subject seated in upright position with feet fully resting on the floor. The elbow flexed to 90 degrees with the arm adducted at side and forearm neutral position. For lateral pinch, the pinch meter was placed between the radial side of index finger and thumb. Subject was instructed to pinch as hard as possible. To measure Pulp to pulp strength, the pinch meter was placed between pulp of the thumb and pulp of the index finger; 3 repetitions were taken and mean of three was considered. For the assessment of Reaction Time, ruler drop test was used,50 $\mathrm{cm}$ scale used for the same. The subject was seated with back supported and hand tested was placed on the table with free movement of wrist joint. The ruler was held by the therapist so that the zero mark was at the level of subject's index finger before the prior warning the ruler was 
Roopa Desai et.al. Association of age with hand grip strength, pinch strength and reaction time in gynaecologists and obstetricians.

dropped and subject was instructed to hold it. The score of reaction time in $\mathrm{cm}$ was converted into seconds using the formula ${ }^{5}$ $\mathrm{d}=\mathrm{vt}+1 / 2 \mathrm{at} \mathrm{t}^{2}$.

\section{Statistical Analysis}

Statistical analysis of collected data was carried out for 30 samples. The data entered in excel sheet was summarized using descriptive statistics for mean and standard deviation. Pearson's correlation method was used to evaluate the correlation.

\section{RESULT}

The total 30 subjects were assessed in this study. The average of right and left side for hand grip strength, lateral pinch, pulp to pulp strength and reaction time reading were taken.

There was positive but low correlation of age with grip strength and hand pinch strength, as stated in graphs from 1.1 to 1.6. It means that with increase in age of Gynaecologists, grip strength and pinch strength was maintained.

There was negative correlation of age with reaction time in Gynaecologists and Obstetricians, as stated from graph 1.7, 1.8, which means that, with aging of Gynaecologists, reaction time was decreased.

Association of age with right hand grip strength
\begin{tabular}{|l|l|l|l|}
\hline $\mathbf{N}$ & DF & r & p \\
\hline 30 & 28 & 0.286 & 0.1256 \\
\hline
\end{tabular}

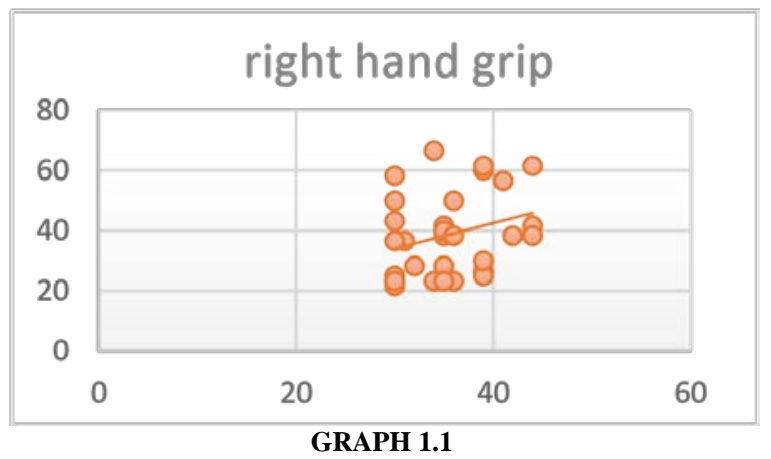

\begin{tabular}{|l|l|l|l|}
\hline $\mathbf{n}$ & Association of age with left hand grip strength \\
\hline 30 & 28 & r & p \\
\hline
\end{tabular}

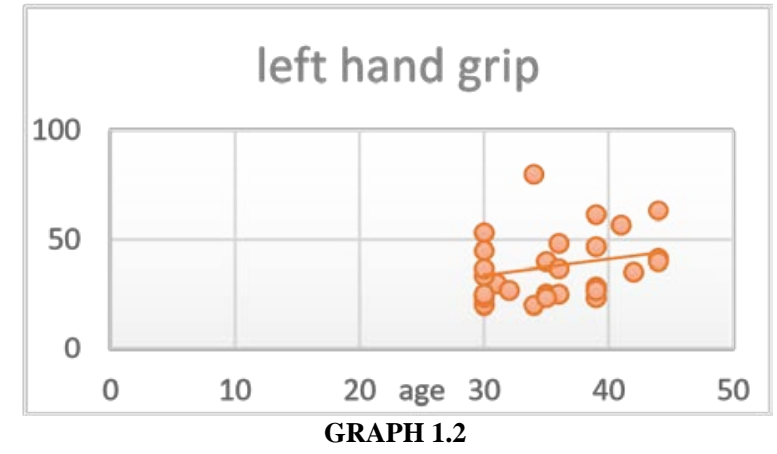

Association of age with right hand key pinch strength

Association of age with right hand key pinch strength
\begin{tabular}{|l|l|l|l|}
\hline n & DF & r & p \\
\hline 30 & 28 & 0.284 & 0.1275 \\
\hline
\end{tabular}
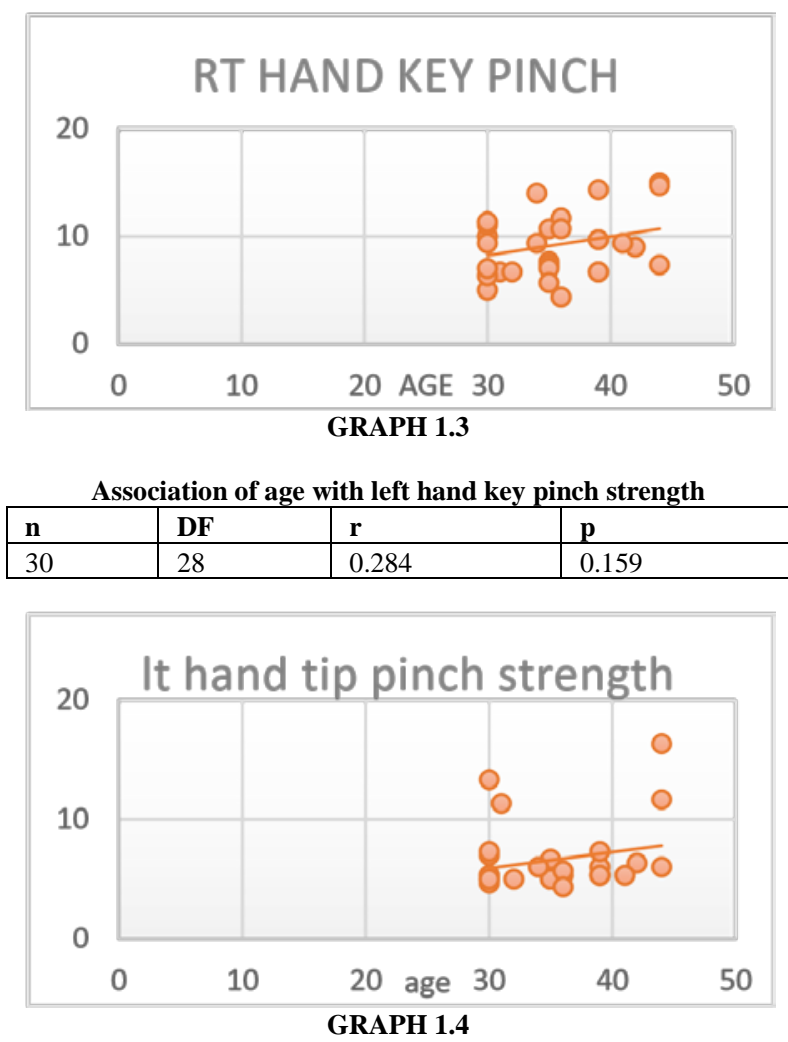

Association of age with right hand tip pinch strength

\begin{tabular}{|l|l|l|l|}
\hline \multicolumn{4}{|c|}{ Association of age with right hand tip pinch strength } \\
\hline 30 & DF & R & p \\
\hline 28 & 0.202 & 0.2831 \\
\hline
\end{tabular}

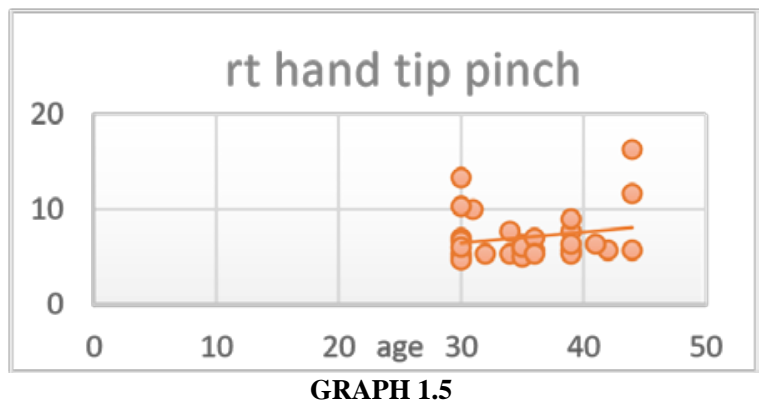

Association of age with left hand tip pinch strength

\begin{tabular}{|l|l|l|l|}
\hline $\mathbf{N}$ & DF & $\mathbf{R}$ & $\mathbf{P}$ \\
\hline 30 & 28 & 0.223 & 0.2353 \\
\hline
\end{tabular}


Roopa Desai et.al. Association of age with hand grip strength, pinch strength and reaction time in gynaecologists and obstetricians.
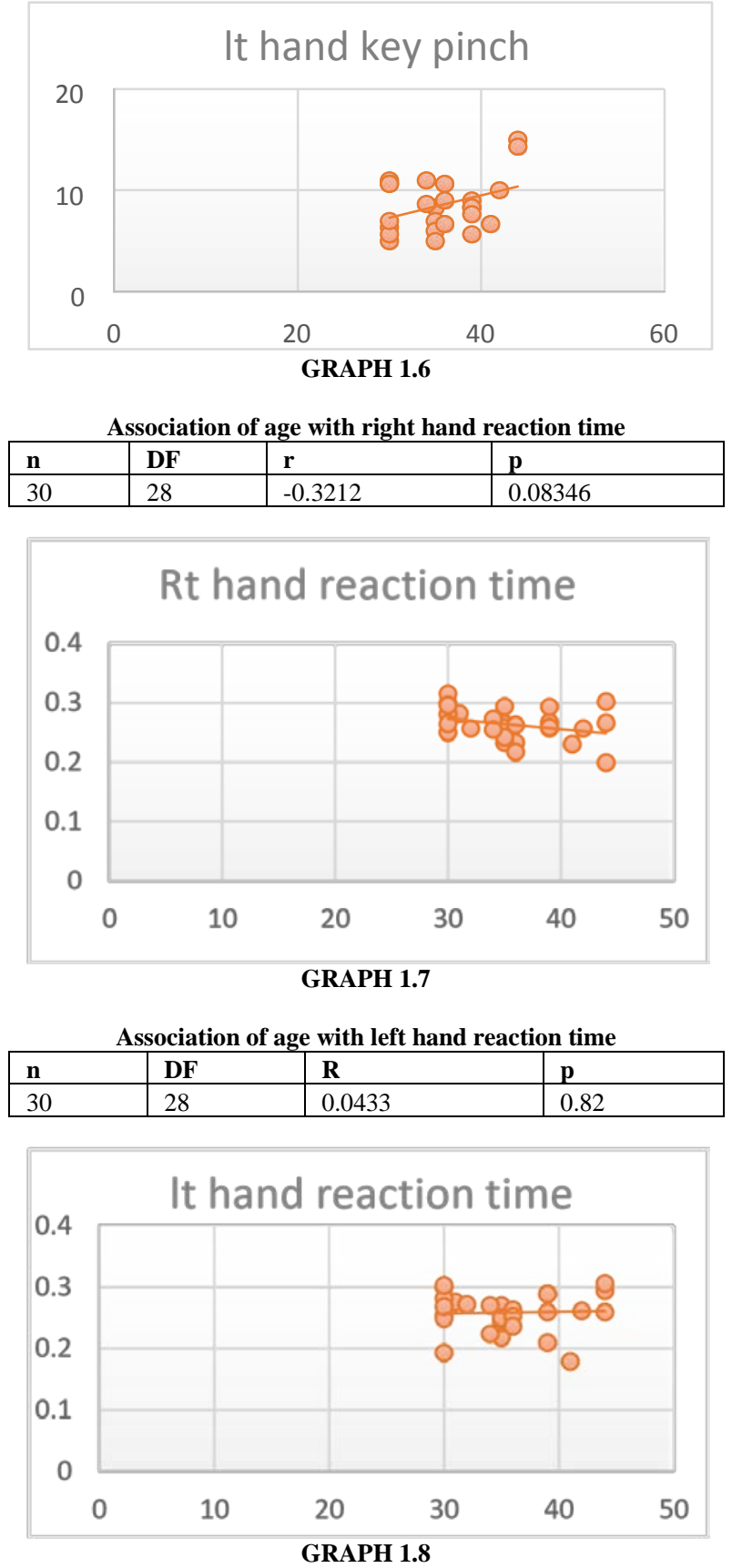

\section{DISCUSSION}

The aim of the study was analysis of association of age with hand grip strength, pinch strength and reaction time of Gynaecologists and Obstetricians. The study had 30 samples, which included 4 male and 26 female surgeons.

Maintaining

musculoskeletal function for the maximal period of time and prevention of musculoskeletal disorders are the most important aspect of healthy ageing surgeons. According to graph 1.1 and 1.2 which concluded that as the age of the gynaecologist increased, the grip strength was maintained. But, since $r<0.3$, there is low correlation between age and grip strength. Performing surgery is both physically and mentally demanding job which leads to multisite pain. The potential explanation for this anomaly may be because of prolonged repetitive contraction of intrinsic muscles, during performing surgeries, must have led to increase on the muscle's strength. According to this study, there is positive correlation with age and grip strength in Gynaecologists and Obstetricians.

Martin JA et al in 2015 conducted a study on age and grip strength predict hand dexterity in adults and explained that with increase in age, there is reduction in muscle mass that cause changes in the properties of muscle recruitment and muscle activation. This in turn has an impact on hand dexterity and hand grip strength. ${ }^{6}$ After 40 years of age, certain age-related changes are seen in muscle properties viz. slowed neural contraction, slowed rate of muscle contraction and increased muscle antagonist co-activation. ${ }^{7,8}$

It is necessary for Surgeons to have precise key pinch strength and tip pinch strength for performing various surgical task such as puncture, dissecting, probing, ligation, stapling, tying knot, suturing. According to graphs 1.3 to 1.6 , which concluded that with increasing age, tip pinch strength and key pinch strength was maintained. The explanation for this is the surgeons have to use dexterity while performing above mentioned surgical task. There is continuous and repetitive movement where in surgeons master in their task which maintains the pinch grip strength as the age increases in surgeons. The $r<0.3$, so there is positive but low correlation with age and pinch grip strength. On the contrary, Marmon et al, conducted a study on Associations among steadiness, strength and hand function across the adult life span in 2011. They observed that increase in age was associated with reduction in 
Roopa Desai et.al. Association of age with hand grip strength, pinch strength and reaction time in gynaecologists and obstetricians.

performance and progressive declines in strength i.e. grip, and pinch grip strength ${ }^{9}$.

Reaction time is the amount of time it takes to respond to a stimulus. It is the ability to respond as quick as possible to a stimulus. This is one of the important components for the Gynaecologists and Obstetricians while performing various surgical tasks. ${ }^{10}$ The graph 1.7 and 1.8, on association of age with reaction time concluded that there is negative correlation with age and reaction time in Gynaecologist.

The study stated that as there is continuous and repetitive stimulus while performing surgeries, their alertness to surrounding stimulus has been increased with increase in age, meaning as the age increased, the time taken by the surgeons to react to a stimulus has been reduced. The reflexes do play a role in reaction time. The explanation for decreased in reaction time in Gynaecologist is as increased age there has been increase in years of experience, which led to same repetitive movement to the surrounding stimulus. So, with increased in practice of Surgeons the movements mimic the reflex, leading decrease in reaction time.

\section{CONCLUSION}

The study concluded that there was low association between age, Grip and Pinch grip strength. There was reduction in reaction time as age increases in Gynaecologists and Obstetricians.

\section{Limitations}

- Small sample size.

- Hours of daily clinical practice was not taken into consideration.

Conflict of Interest: None

Source of Funding: None

Ethical Approval: Approved

\section{REFERENCES}

1. Dalager T, Søgaard K, Boyle E, Jensen PT, Mogensen O. Surgery is physically demanding and associated with multisite musculoskeletal pain: A cross-sectional study. J. Surg. Res. 2019 Aug 1;240:30-9.

2. Adams SR, Hacker MR, McKinney JL, Elkadry EA, Rosenblatt PL. Musculoskeletal pain in gynecologic surgeons. J. Minim. Invasive Gynecol.. 2013 Sep 1;20(5):656-60.

3. Huang J, McGlothlin JD. Ergonomic assessment of obstetrician-gynecologists performing laparoscopic surgery: study of hand size and surgical instruments. J Ergonom. 2017;7:224.

4. Aranha VP, Joshi R, Samuel AJ, Sharma K. Catch the moving ruler and estimate reaction time in children. Indian $\mathrm{J}$ Med Health Sci. 2015 Jan;2(1)

5. Kumar S, Fernandes J. Evaluation of reaction time with variations in levels of alertness in geriatric population: An observational study. Int $J$ Ther Rehabil Res. 2014 Apr 1;3(2):1. doi: 10.5455/ijtrr.00000026

6. Martin JA, Ramsay J, Hughes C, Peters DM, Edwards MG. Age and grip strength predict hand dexterity in adults. PloS one. 2015 Feb 17;10(2):e0117598.

7. Doherty TJ, Vandervoort AA, Taylor AW, Brown WF. Effects of motor unit losses on strength in older men and women.J. Appl. Physiol.1993 Feb 1;74(2):868-74.

8. Norris AH, Shock NW, Wagman IH. Age changes in the maximum conduction velocity of motor fibers of human ulnar nerves. J. Appl. Physiol.1953 Apr 1;5(10): 589-93.

9. Marmon AR, Pascoe MA, Schwartz RS, Enoka RM. Associations among strength, steadiness, and hand function across the adult life span. Med Sci Sports Exerc. 2011 Apr 1;43(4):560-7.

10. Badau D, Baydil B, Badau A. Differences among three measures of reaction time based on hand laterality in individual sports. Sports. 2018 Jun;6(2):45.

How to cite this article: Desai R, Maniar J, Khandare S et.al. Association of age with hand grip strength, pinch strength and reaction time in gynaecologists and obstetricians. Int $J$ Health Sci Res. 2021; 11(12): 157-161. DOI: https:// doi.org/10.52403/ijhsr.20211221 